\title{
Femtogram per Milliliter per Meter Squared
}

National Cancer Institute

\section{Source}

National Cancer Institute. Femtogram per Milliliter per Meter Squared. NCI Thesaurus.

Code C119349.

A unit of concentration equal to femtogram per milliliter, divided by meters squared. 